\title{
Evaluation of an Instrument for Screening Patients at Risk for Chronic Kidney Disease: Testing SCORED (Screening for Occult Renal Disease) in a Portuguese Population
}

\author{
Edgar A.F. de Almeida ${ }^{a-c}$ Carlota Lavinas ${ }^{b}$ Catarina Teixeira ${ }^{a}$ \\ Mário Raimundo ${ }^{a}$ Cristina Nogueira ${ }^{c}$ Maria João Melo ${ }^{a}$ Martina Ferreira ${ }^{c}$ \\ António Sampaio $^{b}$ Inês da Silva Henriques ${ }^{b}$ Cecília Teixeira $^{c}$ Sofia Almeida ${ }^{d}$ \\ António Gomes da Costa ${ }^{a}$ Miguel Leal ${ }^{c}$

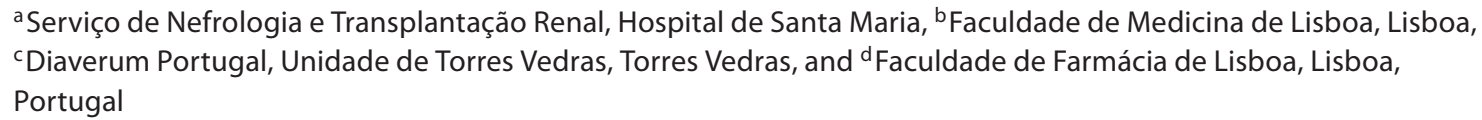

\section{Key Words \\ Chronic kidney disease $\cdot$ Prescreening $\cdot$ SCORED}

\begin{abstract}
Prevalence of chronic kidney disease (CKD) is increasing and CKD has a long asymptomatic phase suitable for screening. SCORED (Screening for Occult Renal Disease) is a prescreening test which has compared favorably with KEEP. We report the results of SCORED testing in subjects attending a World Kidney Day event. After SCORED, subjects were tested for creatinine, urinary albumin and creatinine, and renal ultrasound. Eighty-eight subjects participated (32 men; mean age $59.7 \pm 14.8$ years; $58 \%$ hypertensive and $15.9 \%$ diabetics) of which 60 had a high score for kidney disease. Thirtyeight of $47(80.8 \%)$ subjects that were further evaluated had a high-risk score. All subjects with CKD had a high score (100\% sensitivity). SCORED showed low specificity (24.3\%), but a high negative predictive value (100\%). Including albuminuria in the definition of CKD increased the positive predictive value to $43.6 \%$. In conclusion, SCORED is good for
\end{abstract}

prescreening subjects for CKD in a European population as it captures all patients with CKD. Moreover, in subjects with low risk, the probability of CKD is low. SCORED is useful in alerting the general population and the medical community about the risk factors of CKD.

Copyright $\odot 2012$ S. Karger AG, Basel

\section{Introduction}

Prevalence of chronic kidney disease (CKD) is increasing worldwide [1] and assuming epidemic proportions according to some authors [2]. Portugal has the second highest incidence of patients starting dialysis in Europe [3]. Moreover, prevalence of CKD stages 3-5 in Portugal is $6.1 \%$, which compares to $8.04 \%$ in the National Health and Nutrition Examination Survey (NHANES) 19992004 [4]. Increasing age and increasing cardiovascular risk factors such as diabetes, hypertension and metabolic syndrome may contribute for this high incidence and prevalence of CKD [4]. As CKD remains symptomless for

\section{KARGER \\ Fax +4161306 1234 \\ E-Mail karger@karger.ch}

www.karger.com
(C) 2012 S. Karger AG, Basel

$1420-4096 / 12 / 0356-0568 \$ 38.00 / 0$

Accessible online at: www.karger.com/kbr
Prof. Edgar A.F. de Almeida

Serviço de Nefrologia, Hospital de Santa Maria

Av. Prof Egas Moniz

PT-1639-045 Lisboa (Portugal)

Tel. +35 196868 8477, E-Mail edealmeida@mail.telepac.pt 
most of its natural history, the majority of patients do not start any particular treatment until later stages of the disease. Late referral to nephrologists is a common problem in predialysis care and is related to poor outcomes. Therefore, efforts should be undertaken to screen patients for $\mathrm{CKD}$, in order to start measures of renal protection earlier in the course of the disease.

Screening the whole population for CKD is questionable, but is indicated for high-risk patients. SCORED (Screening for Occult Renal Disease) is a validated tool for screening high-risk patients for CKD [5], with a high sensitivity and high negative predictive value [6]. Using SCORED is inexpensive and is a good tool to select a population at high risk for CKD to perform further analysis of kidney function and imaging, sparing useless exams and inappropriate referral to nephrologists. SCORED has been used in selected populations with high cardiovascular risk and in an unselected population $[6,7]$.

The main purpose of this study was to evaluate the performance of the SCORED questionnaire in a small sample of an unselected Portuguese population which attended a 2011 World Kidney Day event.

\section{Population and Methods}

Subjects attending a 2011 World Kidney Day event in Torres Vedras, Portugal, were invited to participate in this study. The screening was announced in the local media and those wishing to participate were registered and scheduled. The study protocol included collection of demographic data, measuring blood pressure after sitting for $5 \mathrm{~min}$, measuring blood glucose and cholesterol, testing urine for proteins with a urine dipstick and taking the SCORED questionnaire (9 questions) [5]. Two additional questions were included regarding personal and family history of renal disease. Subjects were classified as having high risk for CKD if the SCORED score was 4 or higher, in accordance with the original study [5]. Urine samples were tested for albumin and creatinine and expressed as a urinary albumin-to-creatinine ratio (UACR). Subjects were invited to attend the nephrology outpatient clinic at Hospital de Santa Maria (Lisbon, Portugal) within 3 months to collect blood samples and perform a renal ultrasound. Estimated glomerular filtration rate (eGFR) was calculated using the CKDEPI formula $[8,9]$. CKD was stratified according to eGFR and microalbuminuria, as proposed [10].

All subjects were given indications to preserve renal function as well as a letter to their family practice physician. Subjects with a high score were invited to perform analysis and ultrasound even if they denied further participation in the study. Patients classified as CKD 3 or higher were scheduled for further evaluation at the nephrology department.

Comparisons were done with Student's t test and the $\chi^{2}$ test for continuous and categorical variables, respectively. All statistical analysis was performed using SPSS 16 for Windows ${ }^{\circledR}$.
Table 1. Demographics and clinical characteristics of the study participants

n $\%$

$\begin{array}{lrr}\begin{array}{l}\text { Demography } \\ \text { Total }\end{array} & & \\ \text { Age (years) } & 88 & 100 \\ \quad 18-39 & 10 & 11.4 \\ \quad 40-59 & 27 & 30.7 \\ \quad 60-79 & 46 & 52.3 \\ \quad 79 & 5 & 5.7 \\ \text { Female gender } & 56 & 63.6 \\ \text { Black race } & 2 & 2.3 \\ \text { Basic or primary education } & 41 & 46.6\end{array}$

\section{Clinical condition}

Smoking habits

Alcohol consumption

Hypertension

Diabetes

Dyslipidemia

Knowledge of high urea or creatinine

Previous renal ultrasound

Daily medication

Medical observation in the last 6 months

Serum chemistries in the last 6 months

$6 \quad 6.8$

$40 \quad 45.5$

$51 \quad 58.0$

$14 \quad 15.9$

$54 \quad 61.4$

$7 \quad 8$

$54 \quad 61.4$

$71 \quad 80.7$

$78 \quad 88.6$

$58 \quad 65.9$

The study was sponsored by several institutions, namely Diaverum Portugal, the University of Lisbon School of Medicine, the Nephrology and Renal Transplantation Department - Santa Maria Hospital in Lisbon, City Hall of Torres Vedras District and the Portuguese Society of Nephrology. The study protocol was approved by the Ethics Committee of University of Lisbon School of Medicine and by the Ethics Committee of Diaverum Portugal. All patients gave written informed consent before participating in the study.

\section{Results}

Eighty-eight subjects attended the event (mean age $59.7 \pm 14.8$ years), 56 were women $(63.6 \%)$ and 2 were black (2.3\%; table 1$)$. Hypertension was previously diagnosed in 51 subjects (58\%), diabetes mellitus in 14 (15.9\%), and dyslipidemia in 54 (61.4\%). Moreover, 7 subjects (8\%) knew that they had increased serum creatinine and/or urea. Sixty subjects had SCORED scores of 4 or higher (68.2\%), all but 1 being 50 or more years of age. All diabetics and all subjects with a previous cardiovascular event (cerebrovascular, cardiovascular or peripheral vascular disease) had high-risk scores. Moreover, $94.1 \%$ of subjects with hypertension were classified as high-risk for CKD (table 2). 
Table 2. Comparison between subjects with and without high SCORED scores

\begin{tabular}{|c|c|c|c|c|c|c|c|c|c|c|}
\hline & & \multicolumn{3}{|c|}{ SCORED $\geq 4(\mathrm{n}=60)$} & \multicolumn{3}{|c|}{ SCORED <4 $(\mathrm{n}=28)$} & \multicolumn{2}{|c|}{ Total $(\mathrm{n}=88)$} & $\mathrm{p}$ \\
\hline \multirow{3}{*}{$\begin{array}{l}1 \\
2\end{array}$} & Female gender & 46.6 & 68.3 & 41 & 17.0 & 53.6 & 15.0 & 63.6 & 56 & \multirow{3}{*}{$\begin{array}{l}\text { NS } \\
0.0001\end{array}$} \\
\hline & Age, years & & & & & & & & & \\
\hline & $50-59$ & 12.5 & 18.3 & 11 & 8.0 & 25.0 & 7 & 20.5 & 18 & \\
\hline 3 & Anemia & 3.4 & 5.0 & 3 & 3.4 & 10.7 & 3 & 6.8 & 6 & NS \\
\hline 4 & Hypertension & 54.5 & 80.0 & 48 & 3.4 & 10.7 & 3 & 58.0 & 51 & 0.0000 \\
\hline 5 & Diabetes & 14.8 & 21.7 & 13 & 0.0 & 0.0 & 0 & 14.8 & 13 & 0.007 \\
\hline 6 & Previous MI or stroke & 8 & 11.7 & 7 & 0.0 & 0.0 & 0 & 8.0 & 7 & 0.06 \\
\hline 7 & Cardiac failure & 1.1 & 1.7 & 1 & 0.0 & 0.0 & 0 & 1.1 & 1 & NS \\
\hline
\end{tabular}

Table 3. Stratification of CKD according to low- or high-risk subjects

\begin{tabular}{|c|c|c|c|c|c|c|c|}
\hline \multirow[t]{2}{*}{$\begin{array}{l}\text { CKD } \\
\text { stages }\end{array}$} & \multirow[t]{2}{*}{ eGFR } & \multicolumn{2}{|c|}{$\begin{array}{l}\text { Total } \\
(\mathrm{n}=47)\end{array}$} & \multicolumn{2}{|c|}{$\begin{array}{l}\text { SCORED }<4 \\
(n=9)\end{array}$} & \multicolumn{2}{|c|}{$\begin{array}{l}\text { SCORED } \geq 4 \\
(\mathrm{n}=38)\end{array}$} \\
\hline & & $\%$ & $\mathrm{n}$ & $\%$ & $\mathrm{n}$ & $\%$ & $\mathrm{n}$ \\
\hline 0 or 1 & $\geq 90$ & 22.0 & 10 & 8.5 & 4 & 12.7 & 6 \\
\hline 2 & $60-89$ & 56.1 & 27 & 10.6 & 5 & 46.8 & 22 \\
\hline 3 & $30-59$ & 22.0 & 10 & 0.0 & 0 & 21.2 & 10 \\
\hline
\end{tabular}

Table 4. Cross-tabulation of subjects with high and low SCORED with CKD defined as eGFR $<60 \mathrm{ml} / \mathrm{min}$

\begin{tabular}{rrrrrr}
\hline \multicolumn{3}{c}{ CKD $(\mathrm{eGFR}<60)$} & & \\
\cline { 2 - 4 } & yes & no & total & \\
\hline SCORED high-risk & 10 & 28 & 38 & $26.3 \%$ & PPV \\
low-risk & 0 & 9 & 9 & $100 \%$ & NPV \\
\cline { 2 - 4 } & 10 & 37 & 47 & & \\
\hline & $100 \%$ & $24.3 \%$ & & & \\
& sensitivity & specificity & & \\
\hline
\end{tabular}

$\mathrm{PPV}=$ Positive predictive value; $\mathrm{NPV}=$ negative predictive value.
eGFR, Proteinuria and UACR

Forty-seven subjects (53.4\%; mean age $62.4 \pm 14.6$ years; 33 women) had a further evaluation in the nephrology outpatient clinic that included testing for serum creatinine and UACR, and a renal ultrasound. Their mean eGFR was $75.4 \pm 21.1 \mathrm{ml} / \mathrm{min} / 1.73 \mathrm{~m}^{2}$. Stratification of the subjects in eGFR stages revealed that none had stage 4 or stage 5 CKD, 10 (21.2\%) had CKD stage 3, 27 (57.4\%) had CKD stage 2 , and $10(21.2 \%)$ had CKD stage 1 or no disease. Thirty-three percent of the subjects stratified as CKD stage 3 were previously unaware of their condition. Overall, 38 of the subjects attending the follow-up study had a high-risk score (80.8\%). All subjects with CKD stage 3 were correctly classified as 'high-risk' patients with the SCORED tool; in contrast, 6 of 10 subjects with minimal disease were misclassified as 'high-risk' patients (table 3). When defining CKD as an eGFR $<60 \mathrm{ml} /$ $\mathrm{min} / 1.73 \mathrm{~m}^{2}(10 / 47 ; 21.2 \%)$, the SCORED questionnaire showed $100 \%$ sensitivity but a specificity of only $24.3 \%$, with a positive predictive value of only $26.4 \%$ but a negative predictive value of $100 \%$ (table 4). After including UACR in the definition, more subjects were considered to have CKD (19/47; 40.4\%). The sensitivity of the SCORED questionnaire lowered to $89.5 \%$ and the specificity to $21.4 \%$, while the positive predictive value increased to $43.6 \%$ and the negative predictive value decreased to $75.0 \%$ (table 5). Two subjects with a SCORED of less than 4 were misclassified as not having CKD; they were included because of the presence of UACR and both had a 
Table 5. Cross-tabulation of subjects with high and low SCORED with CKD defined as eGFR $<60 \mathrm{ml} / \mathrm{min}$ or UACR $>30 \mathrm{mg} / \mathrm{g}$

\begin{tabular}{|c|c|c|c|c|c|}
\hline & \multicolumn{3}{|c|}{$\begin{array}{l}\text { CKD } \\
(\mathrm{eGFR}<60 \text { or } \mathrm{UACR}>30)\end{array}$} & & \\
\hline & yes & no & total & & \\
\hline \multirow{4}{*}{$\begin{aligned} \text { SCORED high-risk } \\
\text { low-risk }\end{aligned}$} & 17 & 22 & 39 & $43.50 \%$ & PPV \\
\hline & 2 & 6 & 8 & $75 \%$ & NPV \\
\hline & 19 & 28 & 47 & & \\
\hline & $\begin{array}{l}89.5 \% \\
\text { sensitivity }\end{array}$ & $\begin{array}{l}21.4 \% \\
\text { specificity }\end{array}$ & & & \\
\hline
\end{tabular}
value.

$\mathrm{PPV}=$ Positive predictive value; $\mathrm{NPV}=$ negative predictive

family history of renal disease. Therefore, in our sample the probability of having CKD in a subject with aSCORED score $<4$ was $4.2 \%$.

\section{Discussion}

Although the prevalence of CKD is high, screening the general population is not recommended. Despite the long preclinical phase and the potential serious consequences, screening for CKD in the general population is limited by the fact that stratification based on eGFR is associated with an overestimation of the prevalence of patients with CKD stage 3. Moreover, there is no evidence that patients with CKD stages 1 or 2 will progress to later stages and will eventually need dialysis or transplantation. In fact, in a 5.5-year longitudinal follow-up study only $3.1 \%$ of patients in stages 2-4 progressed to renal replacement therapy, while $24.9 \%$ died [11]. Therefore, screening for CKD in subjects with no risk factors may be associated with unnecessary anxiety and consumption of resources.

Bang et al. [5] proposed an instrument for selecting patients more suitable for screening for renal disease. The SCORED questionnaire is a simple checklist that subjects can apply themselves before submitting to the screening procedures. Its merits include alerting individuals, general practitioners and other clinicians to risk factors for CKD $[5-7,12]$. The SCORED questionnaire was developed from the NHANES 1999-2002 and validated in a combined cohort of the Atherosclerosis Risks in Communities (ARIC) and Cardiovascular Heart Study (CHS), and NHANES 2003-2004 [12]. The performance of the
SCORED questionnaire was evaluated in selected patients, either community samples or clinical settings $[6$, $7,11-13]$.

In the current study, unlike other evaluations that retrospectively applied the questionnaire to databases, we used the SCORED questionnaire prospectively in an unselected population attending a 2011 World Kidney Day event. The percentage of subjects with a high-risk score in our study population is higher than in the NHANES or ARIC studies (68.2\% vs. $51 \%$ in ARIC/CHS and $40 \%$ in NHANES 2003-2004), which may reflect the fact that people attending the 2011 World Kidney Day event are more concerned about kidney disease [12]. Our results confirmed the high sensitivity of the SCORED questionnaire, which detected all subjects with CKD. Conversely, the specificity was low meaning that a substantial number of subjects will be tested for CKD despite not being affected. Nevertheless, the negative predictive value is high, which means that a low score will discard with high certainty the presence of CKD.

In early stages, the definition of CKD depends not only on eGFR evaluation, but also on findings that may anticipate progression of kidney disease, such as proteinuria or renal cysts in patients with a family history of Autosomal Dominant Polycystic Kidney Disease [1], and inclusion of UACR in CKD criteria has recently been proposed [10]. In our study population, it lowered the sensitivity of the SCORED questionnaire because 2 subjects with proteinuria may not be aware of this finding and answered 'no' in the question about proteinuria. Interestingly, both had a family history of kidney disease, but this question is not computed in SCORED. We speculate whether the inclusion of an additional question in the questionnaire will improve the performance of the SCORED score.

We acknowledge some limitations of our study. Firstly, the sample size is small, which could limit the interpretation of results. Nevertheless, to the best of our knowledge this is the first study that applied the SCORED score prospectively and our results are consistent with the larger studies that applied it retrospectively in other populations $[6,7,11-13]$. Another limitation, which is common to other studies, is the fact that the definition of CKD depends on the persistence of the findings during a 3-month period. We were unable to fulfill this criterion, which can lead to an overestimation of CKD prevalence. To reduce the overestimation of CKD in subjects with eGFR $>60$ $\mathrm{ml} / \mathrm{min} / 1.73 \mathrm{~m}^{2}$, we used the CKD-EPI equation instead of the MDRD equation.

Screening is applicable if the disease is serious, if treatment given before symptoms is more beneficial than after 
symptoms (e.g. dialysis) and if the disease is highly prevalent. All these conditions are fulfilled in CKD. The utility of a prescreening test is higher in situations in which the screening test is invasive or expensive. Screening tests for CKD involve determination of serum creatinine, urine dipstick examination and a renal ultrasound; serum creatinine and urine dipstick are routinely performed by most physicians in general practice. The costs of these exams, although relevant, are diluted in the costs of a general check-up. Therefore, in our view, the value of a prescreening test is to increase the awareness of the risk factors for CKD both by physicians (namely primary care physicians) and the general population. The SCORED questionnaire could be used by primary care physicians to evaluate the need for testing for CKD before patients are referred to nephrologists.

In conclusion, our study evaluated SCORED questionnaire in a European population and the results suggest that it is a good tool as a prescreening test for CKD with a high sensitivity and high negative predictive value.

\section{Acknowledgements}

We wish to thank Diaverum Portugal, Câmara Municipal de Torres Vedras, Hospital de Santa Maria, and Faculdade de Medicina de Lisboa for supporting the 2011 World Kidney Day event.

\section{References}

1 Levey AS, Coresh J: Chronic kidney disease. Lancet 2012;379:165-180.

$\checkmark 2$ Coresh J, Stevens LA, Levey AS: Chronic kidney disease is common: what do we do next? Nephrol Dial Transpl 2008;23:1122-1125.

3 ERA-EDTA registry report 2009. www.eraedta-reg.org/.

4 Vinhas J, Gardete-Correia L, Boavida JM, Raposo JF, Raposo AM, Fona MC, et al: Prevalence of chronic kidney disease and associated risk factors, and risk of end-stage renal disease: data from PREVADIAB study. Nephron Clin Pract 2011;119:c35-c40.

5 Bang H, Vupputuri S, Shoham DA, Klemmer PJ, Falk RJ, Mazumdar M, et al: Screening for Occult Renal Disease (SCORED): a simple prediction model for chronic kidney disease. Arch Intern Med 2007;167:374-381.
6 Bang $\mathrm{H}$, Mazumdar M, Newman G, Bomback AS, Ballantyne CM, Jaffe AS, et al: Screening for kidney disease in vascular patients: Screening for Occult Renal Disease Experience (SCORED). Nephrol Dial Transpl 2009;24:2452-2457.

-7 Kwon KS, Bang H, Bomback AS, Koh DH, Yum JH, Lee JH, et al: A simple prediction score for kidney disease in the Korean population. Nephrology 2012;17:278-284.

-8 Stevens LA, Schmid CA, Zhang YL, et al: Development and validation of GFR-estimating equations using diabetes, transplant and weight. Nephrol Dial Transpl 2010;25:449457.

$\checkmark 9$ Levey AS, Stevens LA, Schmid CH, et al: A new equation to estimate glomerular filtration rate. Ann Intern Med 2009;150:604612.

$\checkmark 10$ Levey AS, de Jong PE, Matsushita K, et al: The definition, classification and prognosis of chronic kidney disease: a KDIGO Controversies Conference report. Kidney Int 2011; 80:17-28.
1 Keith DS, Nichols GA, Gullion CM, Brown JB, Smith DH: Longitudinal follow-up and outcomes among a population with chronic kidney disease in a large managed care organization. Arch Intern Med 2004;164:659663.

12 Bang H, Mazumdar M, Kern LM, Shoham DA, August PA, Kshirsargar AV: Validation and comparison of a novel screening guideline for kidney disease: KEEPing SCORED. Arch Intern Med 2008; 168; 432-436.

13 Lucas SM, Nuss G, Stern J, et al: The Screening for Occult Renal Disease (SCORED) values is associated with a higher risk of having or developing chronic kidney disease in patients treated for small, unilateral renal masses. Cancer 2008;113:2681-2686. 